\title{
Erstuntersuchung, bildgebende Verfahren und Entscheidungsfindung bei Azetabulumfrakturen
}

\author{
Jens Stecher, Volker Kammermeier, Ulrich Holz
}

\section{Zusammenfassung}

Das Leitsymptom der Azetabulumfraktur ist die schmerzhafte Bewegungseinschränkung des Hüftgelenkes. Die Diagnose wird anhand der Röntgenübersichtsaufnahme gestellt. Die weitere Beurteilung und Klassifikation erfolgt anhand der axialen Computertomographie. Dreidimensionale Rekonstruktionen verbessern Übersicht und Anschaulichkeit und erleichtern auch dem weniger Erfahrenen die Entscheidung hinsichtlich der Therapieform. Indikationen zur konservativen Therapie mit suprakondylärer Femurextension sind nicht oder wenig verschobene Frakturen, sowie Frakturen außerhalb der Hauptbelastungszone des Azetabulums. Hüftkopfluxationen, Hüftkopffrakturen und eingeklemmte intraartikuläre Fragmente bedürfen einer notfallmäßigen operativen Intervention. Dislozierte Frakturen und Inkongruenzen des Gelenkes werden frühzeitig rekonstruiert und stabilisiert. Nicht rekonstruierbare Azetabulumfrakturen werden entweder primär oder sekundär endoprothetisch versorgt.

\section{Einleitung}

Azetabulumfrakturen treten in der Regel im Rahmen eines Hochenergietraumas mit entsprechender Mehrfachverletzung auf. Sie entstehen in der Mehrzahl der Fälle durch indirekte Traumen. Dabei wird die Gewalt über den Femurkopf auf die Hüftpfanne übertragen. Typisch ist die „dashboard“-Verletzung bei PKW-Unfällen mit Anprall des gebeugten Knies gegen das Armaturenbrett und Gewaltübertragung über die gebeugte Hüfte auf den hinteren Pfeiler des Azetabulums. Weitere indirekte Mechanismen sind die seitliche Krafteinwirkung über den Trochanter major und die axiale Stauchung bei gestrecktem Hüftgelenk. Sehr selten sind direkte Traumen wie die Pfählungsverletzung.

\section{Erstuntersuchung}

Die Unfallanamnese des wachen Patienten gibt Hinweise auf die Azetabulumverletzung. Bei der Untersuchung ist die schmerzhafte Bewegungseinschränkung

OP-JOURNAL 2003; 19: 156-160

(c) Georg Thieme Verlag Stuttgart · New York des Hüftgelenkes das Leitsymptom. Die Luxation oder Subluxation des Hüftkopfes führt zu einer Verkürzung und einer federnden Fixierung des Beines.

Bei nicht ansprechbarem Patienten können die klinischen Hinweise fehlen oder durch weitere Verletzungen kaschiert sein. Dem allgemeinen Polytrauma-Algorithmus folgend sind neben der obligaten Sicherung von Kreislauf und Atemfunktion die Erkennung von eventuellen Schädel-Hirn-, Wirbelsäulen- und Abdominalverletzungen vorrangig.

Die geschlossene Azetabulumfraktur stellt in der Regel keine akut vitale Bedrohung für den Verletzten dar, da sie isoliert nur selten schwere Blutungen verursacht.

In Kombination mit Beckenringinstabilitäten steigt das Risiko für eine kreislaufaktive Gefäßverletzung erheblich an. Eine Ausnahme stellt die seltene offene Azetabulumfraktur dar, bei der eine Blutungskomplikation klinisch führen kann.

Die Erhebung des neurovaskulären Status ist bei der körperlichen Untersuchung obligat, auch wenn ein peripheres Pulsdefi- zit durch begleitende Gefäßverletzungen selten ist. Nervenverletzungen durch Fragmentdislokation oder Hüftkopfluxation finden sich häufiger. Insbesondere der N. ischiadicus in seinem peronealen Anteil ist durch seinen exponierten Verlauf über den hinteren Pfannenrand in 12 bis $38 \%$ der Azetabulumfrakturen betroffen $[1,7]$.

Typische Begleitverletzungen

- Beckenring-Instabilitäten

- Hüftkopfluxation/-fraktur

- Frakturen der unteren Extremitäten

- Mehrfachverletzung/Polytrauma

- Morel-Lavallé-Läsion
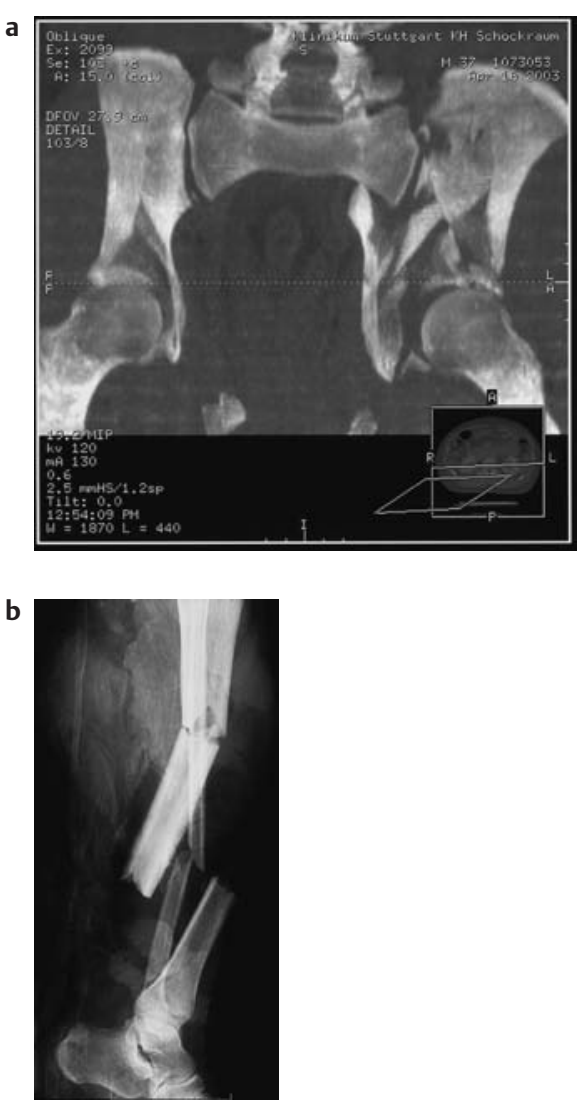

Abb.1a,b Komplexe Becken- und Azetabulumfraktur. Unterschenkeltrümmerfraktur mit nicht rekonstruierbaren Weichteilen. 


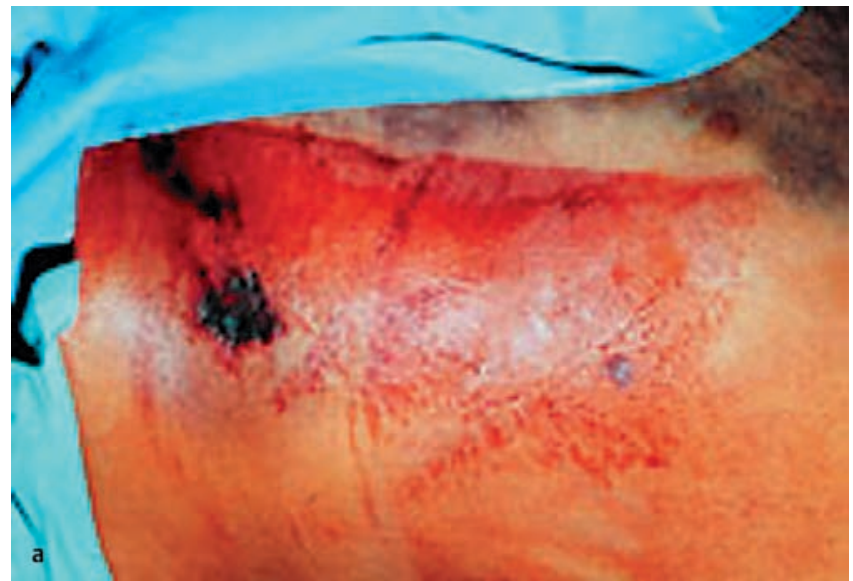

Abb. 2a, b MorelLavallé-Läsion. Chirurgische Revision.

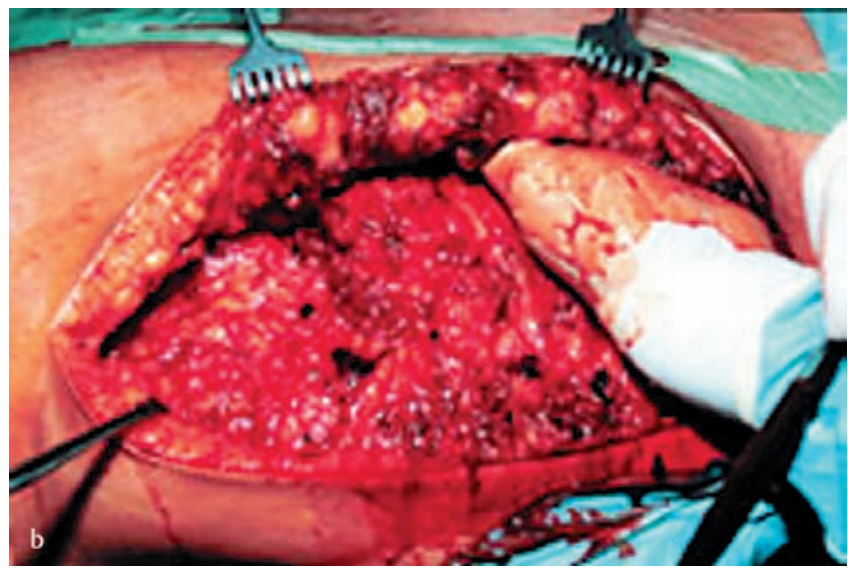

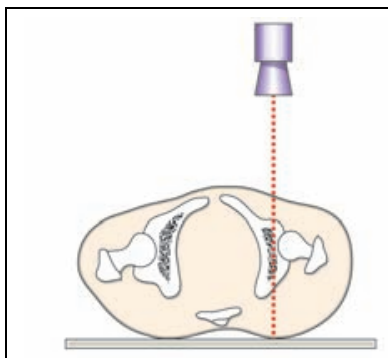

a

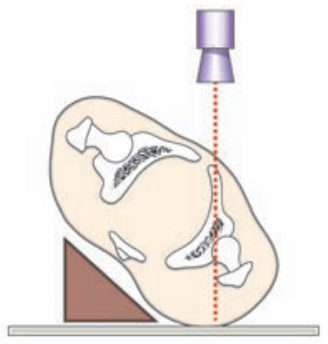

Ala-Aufnahme

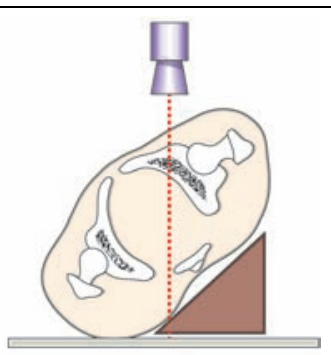

Obturator-Aufnahme b

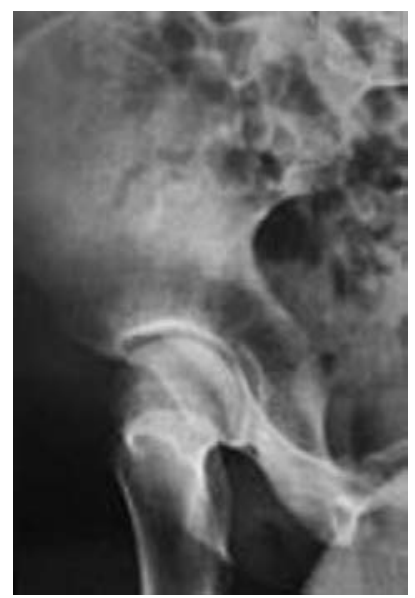

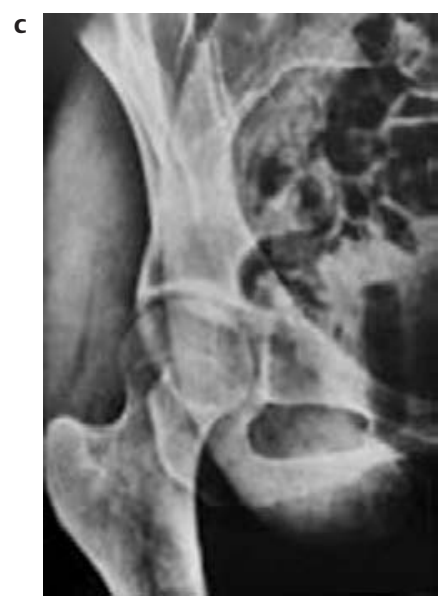

Azetabulumfrakturen treten in der Mehrzahl begleitet von weiteren Verletzungen des Beckenringes und der unteren Extremitäten auf. Je nach Unfallmechanismus gilt dem Ort der Gewalteinwirkung besondere Aufmerksamkeit: Die Kniegelenksregion bei der „dashboard“-Verletzung, der (Rück-)Fluss bei axialer Stauchung, die Trochanterregion bei seitlichem Aufprall. Bei allen indirekten Verletzungen ist der Hüftkopf besonders exponiert.

Eine Hüftkopf-Luxation und/oder eine Hüftkopffraktur bedarf der notfallmäßigen Behandlung.

Bei seitlicher Gewalteinwirkung über die Trochanterregion kann ein geschlossenes Dekollement der Weichteile resultieren mit Ausbildung eines Hämatoms und einer Fettgewebsnekrose. Diese so genannte Morel-Lavallé-Läsion hat ein hohes sekundäres Infektionsrisiko und bedarf daher der chirurgischen Ausräumung und Drainage.

Zur Vervollständigung der klinischen Untersuchung gehört wie bei allen Beckenverletzungen die manuelle - und gegebenenfalls endoskopische - rektale und vaginale Untersuchung. Bei Verletzung von Rektum oder Vagina ist die Azetabulumfraktur als offene Fraktur zu definieren.

\section{Bildgebende Verfahren}

\section{Konventionelles Röntgen}

Die Beurteilung und Klassifikation von Azetabulumfrakturen ist schon aufgrund der komplexen Anatomie der Hüftgelenkspfanne schwierig. Eine exakte Analyse der Verletzung ist aber entscheidend für die Festlegung der Behandlung. Neben der Entscheidung für oder gegen die operative Therapie ist der operative Zugang anhand der präoperativen Diagnostik festzulegen.

Letournel und Judet haben mit ihren $\mathrm{Ar}$ beiten $[3,4,5,6]$ grundlegend zum Verständnis der Azetabulumfrakturen beigetragen. Die von ihnen entwickelte Klassifikation beruht auf der Analyse konventioneller Röntgenbilder: Beckenübersichtsaufnahme, Ala- und Obturatoraufnahme. Bei der Obturator-Aufnahme, auch vordere schräge Judet-Aufnahme genannt, wird die betroffene Hüfte um ca. $45^{\circ}$ angehoben. Bei der Ala-Aufnahme, auch hintere schräge Judet-Aufnah- 


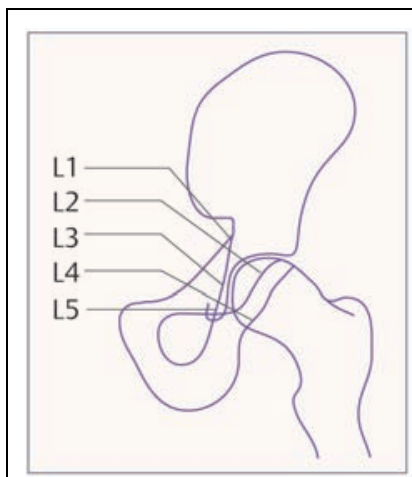

A.p. Projektion

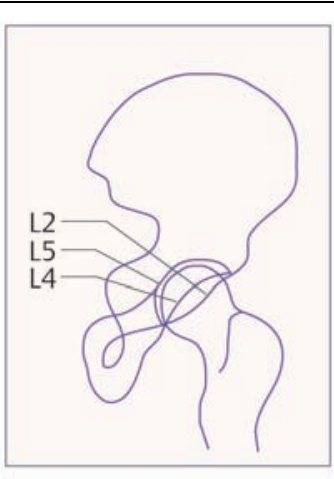

Ala-Aufnahme

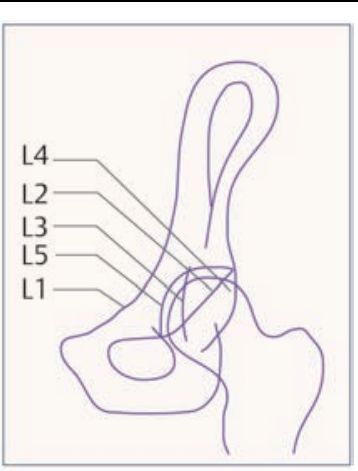

Obturator-Aufnahme

Abb.4 Von Letournel beschriebene Landmarken L1 Ilioinguinale Linie (vorderer Pfeiler) L2 Außenrand vorderer Pfeiler L3 Ilioischiale Linie (hinterer Pfeiler) L4 Außenrand hinterer Pfeiler L5 Tropfenzeichen (Tear-drop).

me genannt, wird die gesunde Hüfte um ca. $45^{\circ}$ angehoben.

Die Einteilung des Azetabulums in vorderen und hinteren Pfeiler ist die Basis der Frakturklassifikationen. Auf den Röntgenaufnahmen werden diese anatomischen Regionen anhand der von Letournel beschriebenen sechs Landmarken nachvollzogen (s. Abb.4).

\section{Computertomographie}

Ursprünglich zur weitergehenden Diagnostik eingesetzt, ist die Computertomographie heute das entscheidende diagnostische Verfahren zur Beurteilung und Klassifikation der Azetabulumfrakturen. Schon in der axialen Computertomographie sind auch kleine intraartikuläre Fragmente, Impressionen der Hüftpfanne, Trümmerzonen oder Begleitverletzungen des Hüftkopfes sicher darstellbar. Durch die im Gegensatz zum konventionellen Röntgen fehlenden Additionseffekte können die einzelnen Strukturen des anatomisch komplexen Gebildes Azetabulums einfacher identifiziert und beurteilt werden. Auch wenn die klassische Einteilung der Frakturen auf dem konventionellen Röntgen beruht, ist die Klassifikation anhand der axialen Computertomographie besser und - zumindest in der Hand des weniger Erfahrenen - sicherer.

Die technisch deutlich aufwändigeren Rekonstruktionen und Femur-Subtraktionsdarstellungen aus dem CT-Datenmaterial bringen als dreidimensionale Projektionen eine Verbesserung für die Praxis. Erwiesen ist der Profit für den in der Beckenchirurgie weniger Erfahrenen, da die Anatomie und Pathologie der Hüft- pfanne übersichtlich und plastisch dargestellt wird und keine Abstraktion der Summationsbilder erfordert [2]. In der
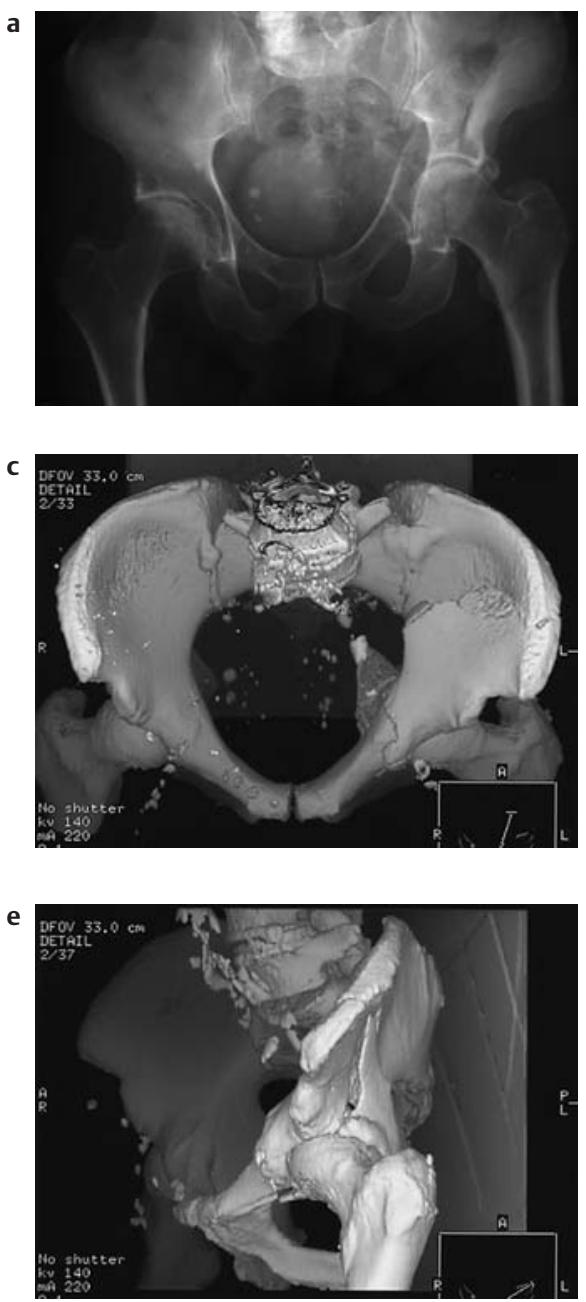

Abb.5a-f Zweipfeilerfraktur. (a) Beckenübersicht. (b) Axiale CT. (c-f) Dreidimensionale Rekonstruktionen.
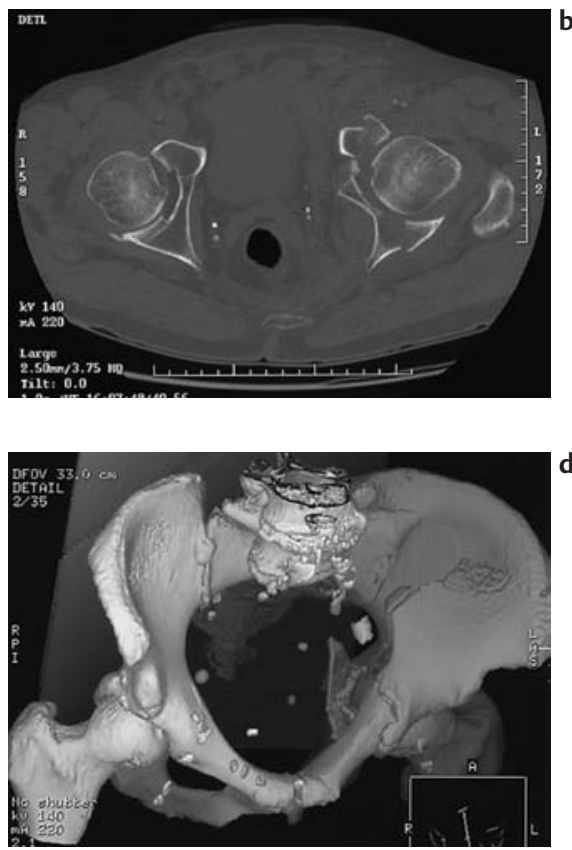

gleichen Arbeit wurde gezeigt, dass in der Beckenchirurgie sehr Erfahrene die Frakturen schon anhand von Beckenübersicht und Schrägaufnahmen beurteilen und klassifizieren können. Allerdings ist auch diese Gruppe wegen im konventionellen Röntgen nicht sicher auszuschließender intraartikulärer Fragmente und Trümmerzonen auf eine zweidimensionale Computertomographie angewiesen, um intraoperative „Überraschungen“ zu vermeiden.

Zeigt sich in der Beckenübersichtsaufnahme eine Azetabulumfraktur, so wird heute selten auf eine CT-Untersuchung verzichtet. Deshalb kann die Ala- und Obturatoraufnahme keine Conditio sine qua non sein.

In Kliniken der Maximalversorgung wird beim Schwerstverletzten der Rumpf zunehmend primär im Spiral-CT untersucht. Hier kann die konventionelle

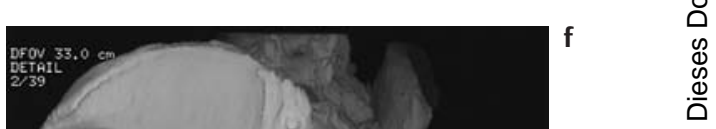



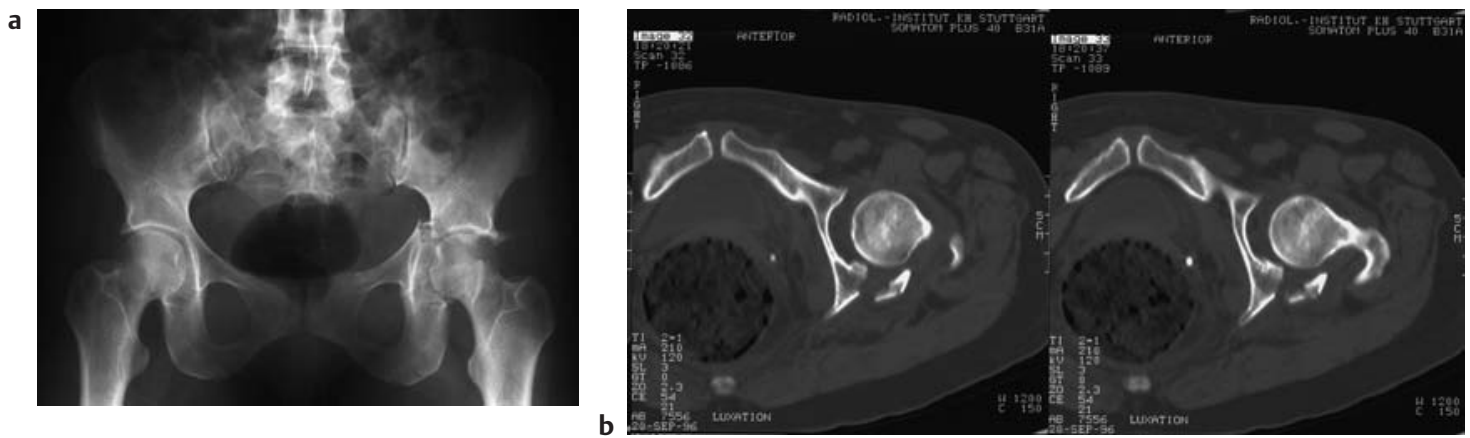

Abb.6a-e Querfraktur mit Hinterwandfraktur. (a) Beckenübersicht. (b) Axiale CT. (c) u. (d) Dreidimensionale Rekonstruktionen. (e) Femursubtraktions-Darstellung.
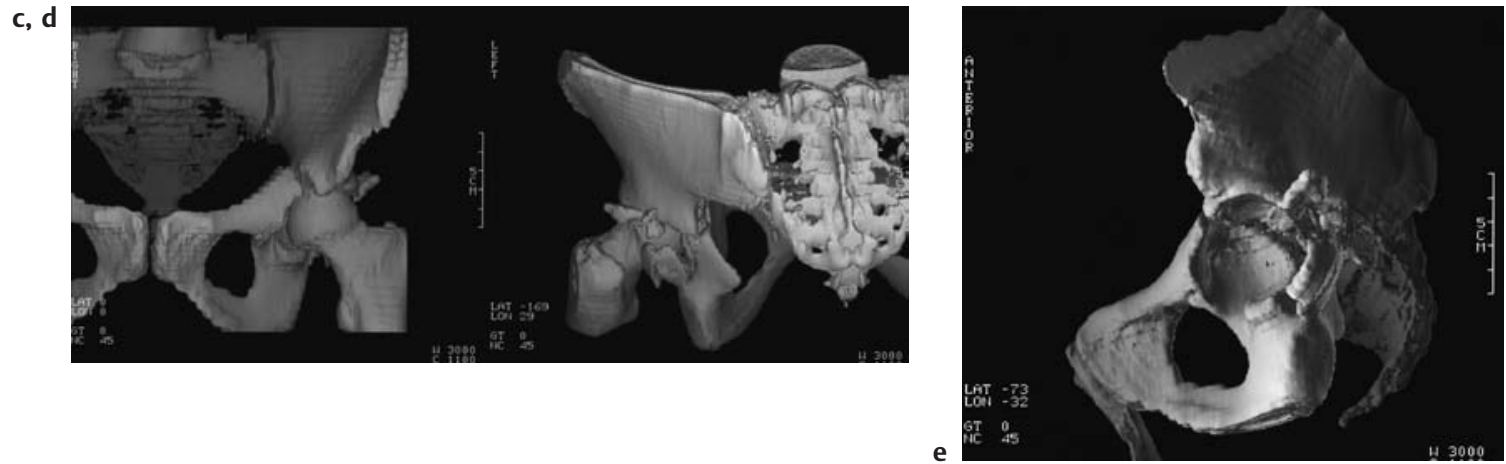

Beckenübersichtsaufnahme durch eine entsprechende Rekonstruktion der a.p.Ebene aus den CT-Daten entfallen.

Wegen der erhöhten Thrombose-Inzidenz sollte bei zuverlegten Patienten mit einer einige Tage zurückliegenden Azetabulumfraktur vor der Operation eine tiefe Beinvenenthrombose ausgeschlossen werden (Duplex-Sonographie, Kontrastmittel-MRT).

\section{Entscheidungsfindung}

Voraussetzung für ein Behandlungskonzept ist die sichere Beurteilung der Azetabulumfraktur und ihre Klassifikation. Weitere Faktoren bei der Entscheidungsfindung sind der Allgemeinzustand des Verletzten, im Falle des Schwerverletzten das Gesamtverletzungsmuster unter Beachtung vorrangig zu behandelnder Verletzungen, vorbestehende Veränderungen des Hüftgelenkes, z.B. eine Koxarthrose, und die Frakturmorphologie. Eine Trümmerzone oder ein intraartikuläres Fragment können die Therapie unabhängig von der Klassifikation beeinflussen.

\section{Konservative Behandlung}

Die operativen Rekonstruktionen von Azetabulumfrakturen sind große Eingriffe und mit einer hohen Morbidität belastet [7]. Bei ernsten Vorerkrankungen oder schweren weiteren Verletzungen, die das allgemeine Operationsrisiko erhöhen, muss gegebenenfalls trotz frakturbedingter Operationsindikation der konservativen Therapie der Vorzug gegeben werden. Auch bei ausgeprägter Osteoporose sollte die frakturbedingte Operationsindikation in den Hintergrund treten.

Die Indikationen zur operativen Therapie der Azetabulumfrakturen sind immer noch im Fluss und werden zum Teil kontrovers diskutiert.

In der Gelenkchirurgie werden Gelenkstufen von bis $\mathrm{zu} 2 \mathrm{~mm}$ toleriert. Im Umkehrschluss ist bei Gelenkfrakturen, die eine so geringe Dislokation aufweisen, die konservative Therapie indiziert. Ebenso sind Frakturen in weniger belasteten Anteilen der Hüftpfanne ohne Operation zu behandeln. Dies betrifft Fraktu-

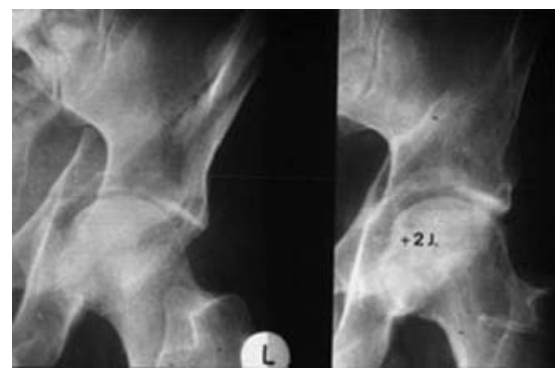

Abb.7 Sekundäre Kongruenz nach Extensionsbehandlung. ren der kaudalen Anteile des vorderen Pfeilers und weit kaudal verlaufende Quer-Frakturen. Kleine ausgesprengte Fragmente, zum Beispiel an der hinteren Pfannenbegrenzung, die die Stabilität des Gelenkes nicht beeinträchtigen, bedürfen ebenfalls keiner operativen Therapie.

Indikationen zur konservativen Behandlung:

- Fraktur-unabhängige Inoperabilität

- nicht oder minimal dislozierte Fraktur mit erhaltener Gelenk-Kongruenz und Gelenk-Stabilität

- Frakturen außerhalb der Hauptbelastungszone:

- Frakturen im kaudalen Anteil des vorderen Pfeilers

- weit kaudal verlaufende Querfrakturen

- kleines Fragment der hinteren Pfannenbegrenzung bei stabilem Gelenk

- gute sekundäre Kongruenz

- schwere Koxarthrose

Bei einem konservativen Therapieansatz über eine Extension ist das Ziel, eine sekundäre Kongruenz durch Ligamentotaxis zu erreichen. Die Frakturfragmente folgen dem Kopf bei der Traktion [8]

Bewährt hat sich für die konservative Therapie eine suprakondyläre Drahtextension mit einem Gewicht, das einem Achtel bis Zehntel des Körpergewichts entspricht. 
Bei Anlage der Extension am Oberschenkel ist eine vorsichtige Beübung von Hüft- und Kniegelenk mit einer Motorbewegungsschiene möglich. Der angegebene zusätzliche Seitzug über einen von lateral in das Trochantermassiv eingebrachten Dübel ist nicht zu empfehlen, da er keine Verbesserung des Repositionsergebnisses erwarten lässt, aber ein erhebliches Risiko für eine Infektion an der Eintrittstelle an der Haut darstellt.

\section{Operative Behandlung}

Die Luxationsstellung des Hüftkopfes und/oder Hüftkopffrakturen bedürfen notfallmäßiger Behandlung.

Sie sind operativ zu versorgen, um eine weitergehende Gelenkschädigung zu vermeiden. Die anderen Azetabulumfrakturen werden früh-elektiv in einem Zeitrahmen bis etwa 20 Tage operativ versorgt. Je früher die Operation erfolgt, desto leichter gelingt die Reposition. Gegebenenfalls wird präoperativ die Operabilität des Patienten verbessert und die Diagnostik vervollständigt. Der Verletzte kann in der Zwischenzeit in die Entscheidungsfindung mit einbezogen werden.

Ziel der operativen Therapie bei Azetabulumfrakturen ist die Wiederherstellung kongruenter Gelenkflächen mit stabiler zentraler Stellung des Hüftkopfes und Übungsstabilität.

Sofern keine der oben aufgeführten allgemeinen Kontraindikationen besteht, ist die operative Therapie bei Frakturen des Azetabulums, die die Hauptbelastungszone betreffen und mehr als $2 \mathrm{~mm}$ disloziert sind, indiziert. Hierunter zählen auch ausgedehnte Impressionsfrakturen der Gelenkfläche, die aufgerichtet und unterfüttert werden müssen.

Indikationen zur operativen Behandlung: dringlich

- Luxation oder Einklemmung des Hüftkopfes

- Instabilität des Hüftgelenkes

- intraartikuläre Fragmente

frühzeitig

- Stufenbildung der Gelenkfläche (>2 mm)

- ausgedehnte Impressionen der Gelenkfläche/Inkongruenz

Die Wiederherstellung der Gelenkfläche ist bei ausgeprägter Koxarthrose per se nicht notwendig. Es ist dennoch die Indikation zur operativen Intervention zu prüfen, um ein stabiles Lager für die

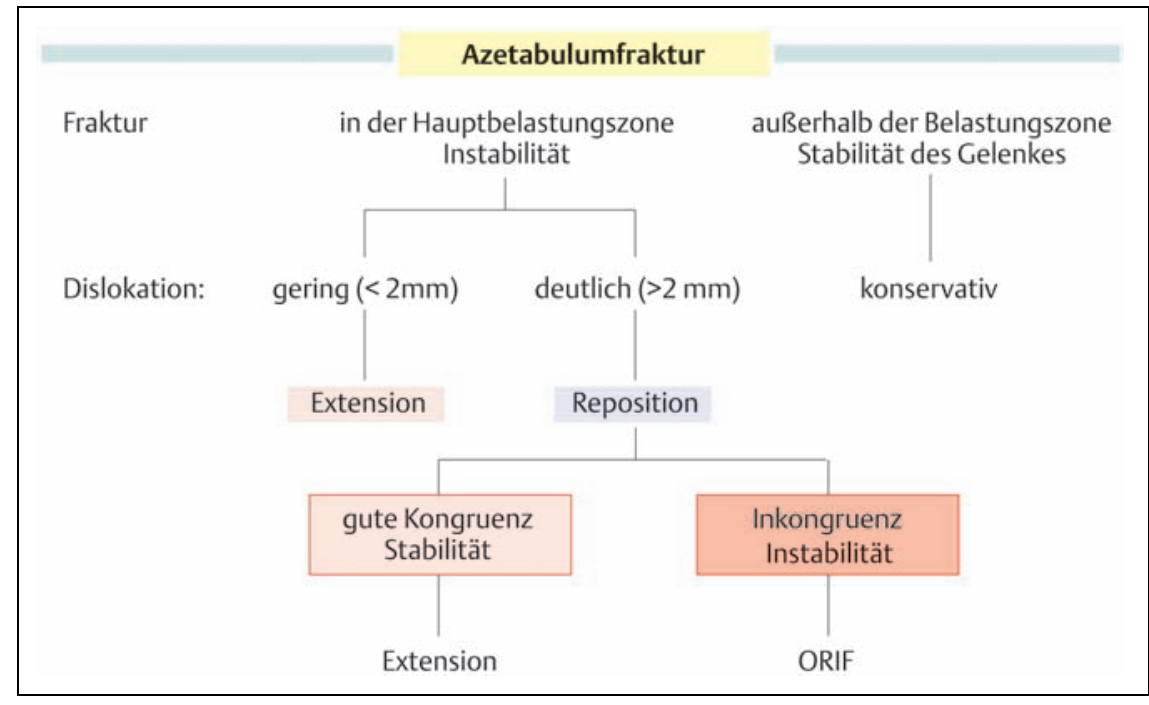

Abb. 8 Algorithmus zur Therapie der Azetabulumfraktur.

sekundäre Implantation einer Hüftgelenkstotalendoprothese $\mathrm{zu}$ schaffen, sofern die primäre Implantation einer Hüftgelenkstotalendoprothese aus frakturmorphologischen Gründen nicht möglich ist. Insbesondere bei stärkerer Dislokation von Fragmenten oder ausgedehnten Defekten erleichtert eine im Aufwand begrenzte Rekonstruktion der Pfannenanatomie die spätere endoprothetische Versorgung.

\section{Schlussfolgerung}

Azetabulumfrakturen treten vorrangig bei Mehrfachverletzten auf und stehen bei der Akutversorgung in der Regel therapeutisch im Hintergrund. Nur bei starker Fragmentdislokation oder im Rahmen einer Beckeninstabilität besteht eine erhöhte Inzidenz von kreislaufrelevanten Blutungen. Zur Erfassung und Klassifikation ist neben der konventionellen oder aus dem CT-Datensatz rekonstruierten Beckenübersicht eine Computertomographie notwendig. Mit der axialen CT können der Frakturtyp und Begleitverletzungen auch vom in der Beckenchirurgie weniger Versierten sicher erkannt werden. Dreidimensionale Rekonstruktion und Femursubtraktionsdarstellung verbessern die Übersicht und Anschaulichkeit, sind aber ebenso wie konventionelle Schrägaufnahmen verzichtbar. Sofern keine Kontraindikationen bestehen, werden um mehr als $2 \mathrm{~mm}$ dislozierte Frakturen in der Hauptbelastungszone des Azetabulums in Narkose reponiert und im Falle unbefriedigender Kongruenz oder Instabilität offen reponiert und intern stabilisiert.

\section{Literatur}

1 Helfet DL, Bartlett CS. Acetabular fractures. In: Rüedi, T.P., W.M. Murphy: AO Principles of Fracture Management. Thieme Stuttgart 2000, S. $415-440$

2 Hüfner T, Pohlemann T, Gänsslen A, Assassi P, Prokop M, Tscherne H: Die Wertigkeit der CT zur Klassifikation und Entscheidungsfindung nach Azetabulumfrakturen. Unfallchirurg 1999; 102: 124-131

3 Judet R, Judet J, Letournel E: Fractures of the acetabulum: classification and surgical approaches for open reduction. J Bone Joint Surg AM 1964; 46: 1615-1646

${ }^{4}$ Letournel E: Les fractures du cotyle. Etude d'une série de 75 cas. J Chir 1961; 82: 47

${ }^{5}$ Letournel E: Acetabulum fractures: classification and management. Clin Orthop 1980; 151: 81-106

${ }^{6}$ Letournel E, Judet R: Fractures of the acetabulum. Springer, Berlin, Heidelberg 1993

7 Pohlemann T, Hüfner T, Gänsslen A: Komplikationen. In: Tscherne, H., T. Pohlemann: Becken und Azetabulum. Springer, Berlin, Heidelberg 1998, S. 433-440

8 Rommens PM, Hessmann MH. Azetabulumfrakturen. Unfallchirurg 1999; 102: 591 - 610

\section{Dr. med. Jens Stecher}

Assistenzarzt

Dr. med. Volker Kammermeier

Leitender Oberarzt

Prof. Dr. med. Ulrich Holz

Direktor der Klinik für Unfall- und

Wiederherstellungschirurgie

Katharinenhospital Stuttgart

Klinik für Unfall- und Wieder-

herstellungschirurgie

Kriegsbergstr. 60

D-70174 Stuttgart 